\title{
An algorithm for the transportation problem with given frequencies
}

\author{
L. Bertazzi, M. G. Speranza \\ Dept. of Quantitative Methods - University of Brescia \\ Corso G. Mameli, 27-I-25122 Brescia, Italy. Tel: 39-30-2988.1. \\ Fax: 39-30-2400925. e-mail: speranza@master.cci.unibs.it \\ W. Ukovich \\ D.E.E.I.-University of Trieste \\ Via A. Valerio, $10-I-34127$ Trieste, Italy. Tel: 39-40-6767135. \\ Fax: 39-40-6763460. e-mail: ukovich@univ.trieste.it
}

\begin{abstract}
In this paper we consider the problem of shipping several products from an origin to a destination, when a discrete set of shipping frequencies is available only, in such a way that the sum of the transportation and inventory cost is minimized. This problem, which is known to be NP-hard, has applications in transportation planning and in location analysis. We derive some dominance rules for the problem solutions which allow to tighten the bounds on the problem variables. Moreover, we present a branch-and-bound algorithm and we evaluate its performance on randomly generated problem instances.
\end{abstract}

\section{Keywords}

Transportation, inventory, dominance rules, branch-and-bound.

\section{INTRODUCTION}

We consider the situation in which each product of a given set is made available at an origin and needed at a destination at a constant rate. A discrete set of available shipping frequencies is given and the shipment is carried out by trucks with given capacity. The problem is to decide how much of each product to ship at each frequency in such a way that the sum of the transportation and inventory cost is minimized.

This problem was introduced in Speranza and Ukovich (1994) together with other models for shipping products from an origin to a destination. Similar models, where the shipping frequencies are allowed to take any continuous value, are considered in Burns et al (1984), Blumenfeld et al (1985), Anily and Federgruen (1990). The problem was shown to be NP-hard in Speranza and Ukovich (1991), where an optimal branch-and- 
bound algorithm was presented. The computational results showed that the algorithm was efficient on problem instances of small/medium size. The more complex problem of shipping products from several origins to a destination, when a given set of shipping frequencies is available, was considered in Bertazzi, Speranza and Ukovich (1994), where different heuristics were presented, based on solving in a first phase a one origin - one destination problem for each of the given destinations, and then on improving the solution through local search techniques.

In this paper, we derive some dominance rules for the solution of the one-origin-onedestination problem. Such rules allow to tighten the bounds on the problem variables. Then we present a new branch-and-bound algorithm in which we apply the dominance rules in order to improve its efficiency and we compare it with the branch-and-bound proposed in Speranza and Ukovich (1991).

The paper is organized as follows. In Section 2 the problem is described and its mathematical programming formulation is given. The dominance rules and their implications are presented in Section 3. In Section 4 the new branch-and-bound is described and the computational results are given in Section 5.

\section{PROBLEM DESCRIPTION AND FORMULATION}

A set of products is made available at an origin and needed at a destination at a constant rate. Trucks, with given capacity, are available for shipping products, and each product can be partly shipped by trucks travelling at different frequencies. Each product can be continuously divided. Two cost factors are considered, namely the transportation and the inventory cost. Shipping products at a high frequency generates low inventory costs but high transportation costs, while shipping at a low frequency generates high inventory costs and low transportation costs. The problem is to decide the fraction of each product which is shipped at each frequency in such a way that the sum of the transportation and the inventory costs is minimized.

The following notation is used.

$I$ : set of products;

$J$ : set of frequency indices;

$n$ : number of frequencies $(=|J|)$;

$i$ : products;

$j$ : index of frequency;

$f_{j}: j$ th frequency, with $j \in J$;

$t_{j}$ : period of frequency $f_{j}\left(=1 / f_{j}\right)$;

$H$ : time horizon (equal to the minimum common multiple of the periods);

$q_{i}$ : rate at which product $i$ is produced at the origin and needed at the destination;

$v_{i}$ : unit volume of product $i$;

$h_{i}$ : inventory cost of one unit of product $i$ per unit time;

$r_{j}$ : capacity of each truck travelling at frequency $f_{j}$;

$c_{j}$ : cost of a single trip of a truck travelling at frequency $f_{j}$; 
$x_{i j}$ : fraction of product $i$ shipped at the frequency $f_{j}$;

$y_{j}$ : number of trucks which travel at the frequency $f_{j}$.

The problem can be formulated as follows.

$$
\begin{aligned}
& \min \sum_{i \in I} \sum_{j \in J} h_{i} t_{j} q_{i} H x_{i j}+\sum_{j \in J} c_{j}\left(H / t_{j}\right) y_{j} \\
& t_{j} \sum_{i \in I} v_{i} q_{i} x_{i j} \leq r_{j} y_{j} \quad j \in J \\
& \sum_{j \in J} x_{i j}=1 \quad i \in I \\
& 0 \leq x_{i j} \leq 1 \quad i \in I, j \in J \\
& y_{j} \geq 0 \quad \text { integer } \quad j \in J .
\end{aligned}
$$

The objective function (1) represents the sum of the inventory cost and the transportation cost on the time horizon $H$. Constraints (2) are capacity constraints, which state that the number of trucks $y_{j}$ must be sufficient to load all the products assigned to frequency $f_{j}$. Constraints (3) impose that all the quantity of product $i$ produced at the origin must be shipped to the destination at some of the given frequencies. The mixed integer programming problem defined through (1)-(5) will be referred to as Problem $\mathcal{P}$.

In the following, we assume that the unit time is chosen in such a way that all $t_{j}$ are integer and that the frequencies are ordered in the decreasing order, that is $f_{1}>f_{2}>$ $\ldots>f_{n}$. Moreover, we assume that all trucks have the same capacity, independently of the frequency, that is that $r_{j}=r, \forall j$, and, without loss of generality, we normalize the capacity to $r=1$. As all trucks have the same capacity, we assume $c_{j}=c, \forall j$.

\section{DOMINANCE CONDITIONS}

In this section we present the most important theoretical results we have obtained for the Problem $\mathcal{P}$. For their formal proofs, we refer to Bertazzi, Speranza and Ukovich (1995).

Dominance is a concept, common in multiobjective optimization, that turns out to be convenient for our problem. In fact, Problem $\mathcal{P}$ can be considered as stemming from an optimization problem with two conflicting objectives: minimize the inventory cost and minimize the transportation cost.

In general terms, a feasible solution for a multiobjective optimization problems is dominated if another feasible solution exists with not worse performance with respect to each objective, and a strictly better performance with respect to at least one objective (cf. for instance Keeney and Raiffa, 1976).

The following result states a general condition guaranteeing that a solution is dominated. 
Lemma 1 A solution $(\bar{x}, \bar{y})$ for Problem $\mathcal{P}$ is dominated if $\exists \alpha \in \Re_{+}$(the set of positive real numbers), $j, k \in J$ such that:

$t_{k}<t_{j}$

$\alpha \leq \bar{V}_{j} / t_{j}$

$\left\lceil\bar{V}_{j}-\alpha t_{j}\right\rceil / t_{j}+\left\lceil\bar{V}_{k}+\alpha t_{k}\right\rceil / t_{k} \leq \overline{y_{j}} / t_{j}+\overline{y_{k}} / t_{k}$

where $\bar{V}_{s}=t_{s} \sum_{i} v_{i} q_{i} x_{i s}$ for $s=j, k$.

It is worth to point out that the above result generalizes the saturation property for Problem $\mathcal{P}$ presented in Speranza and Ukovich (1994):

Corollary 1 Consider an optimal solution $(\bar{x}, \bar{y})$ for Problem $\mathcal{P}$. Suppose $\exists j, k \in J$ such that $j>k, \bar{V}_{j}>0$. Then $\bar{y}_{k}=\bar{V}_{k}$.

As a consequence of the last result, in the sequel we always consider solutions satisfying the saturation condition. Then Lemma 1 yields a more convenient condition for domination:

Theorem 1 A feasible solution $(\bar{x}, \bar{y})$ for Problem $\mathcal{P}$ is dominated if $\exists \alpha \in \Re_{+}, j, k \in J$ such that conditions (6) and (7) of Lemma 1 are satisfied, and

$\hat{c}_{T}=\left\lceil\bar{V}_{j}-\alpha t_{j}\right\rceil+\left\lceil\alpha t_{k}\right\rceil t_{j} / t_{k} \leq \bar{y}_{j}$

Corollary 2 A solution $(\bar{x}, \bar{y})$ for Problem $\mathcal{P}$ is dominated if $\bar{y}_{j} t_{k} / t_{j} \geq 1$ for some $j>k$ with $t_{j} / t_{k}$ integer.

Corollary 2 allows to tighten the bounds for the variables $y_{j}, j>1$, whenever $t_{j}$ admits a proper submultiple in the given set of periods $\left\{t_{j}\right\}$. Let us denote by $J^{\prime} \subseteq J$ the set of indices of the periods which admit a proper submultiple. Then

$y_{j} \leq U B 1_{j}=\min _{k}\left\{t_{j} / t_{k}-1: t_{j} / t_{k}\right.$ is integer $\} \quad j \in J^{\prime}$.

In the case $J^{\prime}=J$, then $y_{j} \leq U B 1_{j}, \forall j$, and the range for $y_{1}$ can be made tighter. In fact,

$y_{1} \geq t_{1}\left(\sum_{i} v_{i} q_{i}-\sum_{j=2}^{n} y_{j} / t_{j}\right) \geq t_{1}\left(\sum_{i} v_{i} q_{i}-\sum_{j=2}^{n} \min \left(U B 0_{j}, U B 1_{j}\right) / t_{j}\right)=L B_{1}$

where $U B O_{j}=\left\lceil t_{j} \sum_{i \in I} v_{i} q_{i}\right\rceil$.

Observe that, in this case $\left(J^{\prime}=J\right)$, the width of the range of the variables does not depend on the volume to be shipped, but on the periods only. Let us emphasize this feature. Since $U B 1_{j} \leq t_{j}-1$, then $y_{1} \geq\left\lceil t_{1}\left(\sum_{i} v_{i} q_{i}-n+1+\sum_{j=2}^{n} f_{j}\right)\right\rceil \geq U B 0_{1}-t_{1}(n-1)$. 
A complete enumeration of the solutions generates a number of solutions not larger than $\left(t_{1}(n-1)+1\right) t_{2} \ldots t_{n}$. This quantity depends on the number and on the values of the periods only, which in practice are quite moderate. Thus, for fixed $n$, such a "brute force" enumeration turns out to be just pseudo-polynomial.

A different kind of bounds for the variables $y_{j}, j>1$ can be inferred from a new domination result, which in turn is another consequence of Theorem 1:

Corollary 3 A solution $(\bar{x}, \bar{y})$ for Problem $\mathcal{P}$ is dominated if $\bar{V}_{j} \geq t_{j}$ for some $j>1$.

As a consequence of the last result, then $\bar{V}_{j}<t_{j}$ in any optimal solution, hence $\left\lceil\bar{V}_{j}\right\rceil=$ $y_{j} \leq t_{j}, \forall j>1$. Furthermore, note that if $y_{j}=t_{j}$, then the above Corollary still applies (i.e. the corresponding solution is still dominated), thus yielding a tighter upper bound for $y_{j}, j>1$, equal to $t_{j}-1$.

Another different dominance rule is given by the following theorem. which states that a solution is dominated if all products travelling at a certain frequency can be shipped by full load trucks at a higher frequency.

Corollary $4 \mathrm{~A}$ solution $(\bar{x}, \bar{y})$ for Problem $\mathcal{P}$ is dominated if $\bar{V}_{j} t_{k} / t_{j}$ is an integer positive number for some $j>k$.

Corollary 4 can be further generalized as follows.

Theorem 2 Given a solution $(\bar{x}, \bar{y})$, if a subset $\bar{J}$ of the frequencies and a frequency $f_{k}$ exist such that $t_{j}>t_{k}, \forall j \in \bar{J}$, and $\sum_{j \in \bar{J}} \bar{V}_{j} t_{k} / t_{j}$ is an integer positive number, then the solution is dominated.

\section{A BRANCH-AND-BOUND ALGORITHM}

In this section we present a branch-and-bound algorithm for the solution of the Problem $\mathcal{P}$. In this algorithm we apply the dominance rules we have derived in Section 3; this algorithm will be compared in Section 5 with the branch-and-bound proposed in Speranza and Ukovich (1991) in order to evaluate its performance.

The branch-and-bound works on a search tree where each level corresponds to a frequency $f_{j}$ and each node $\nu$ represents the vehicles $y_{j}$ used at this frequency. This number of vehicles belongs to a set of values defined in the following way.

We recall that, by the Corollary $3, y_{j}<t_{j} \forall j>1$ and that, in the particular case in which the period $t_{j}$ admits a submultiple in the given set of the periods $\left\{t_{j}\right\}$, by the Corollary $2, y_{j} \leq U B 1_{j}=\min _{k}\left\{t_{j} / t_{k}-1: t_{j} / t_{k}\right.$ is integer $\}$. Furthermore, given a partial solution $\left(\bar{y}_{1}, \ldots, \bar{y}_{j-1}\right), y_{j} \leq U B 2_{j}$ with $U B 2_{j}=\left[\left(\sum_{i} v_{i} q_{i}-\sum_{k=1}^{j-1} \bar{y}_{k} / t_{k}\right) t_{j}\right]$. Therefore, the upper bound for $y_{j}, j>1$, is, in general, $y_{j} \leq \min \left(t_{j}-1, U B 2_{j}\right)$ and, in the particular case in which $t_{j}$ has a submultiple in $\left\{t_{j}\right\}, y_{j} \leq \min \left(t_{j}-1, U B 1_{j}, U B 2_{j}\right)$. The lower bound for $y_{j}$ is $L B 1$ for $j=1$, as defined in (11) and $y_{j}=0$ for all $j>1$.

The tree is explored in the depth first way.

The fathoming criteria are the following. An upper bound $U$ is initially set to the minimum value obtained by solving the heuristics presented in Bertazzi, Speranza and 
Ukovich (1995) on the instance of the problem; this upper bound mantains the best current upper bound during the algorithm.

In each node $\nu$, a lower bound $L_{\nu}$ is calculated by solving the relaxed problem by the Procedure Ext-Greedy presented in Speranza and Ukovich (1991). If the solution of the relaxed problem is integer, this node is fathomed. If $L_{\nu}>U$, the node $\nu$ is fathomed. Furthermore, in each node $\nu$ the dominance rules presented in Section 3 are applied; if the obtained solution is dominated, the node $\nu$ is fathomed. When the number of vehicles $y_{j}$ are fixed in all the level of the tree, if the solution is feasible, the optimal value is calculated and the node $\nu$ is fathomed. If the optimal value is lower than the current upper bound $U$, then $U$ is modified.

\section{COMPUTATIONAL RESULTS}

The branch-and-bound proposed in Speranza and Ukovich (1991) and the new branchand-bound have been implemented in FORTRAN on a Personal computer with an Intel $486 \mathrm{dx} / 66$ processor.

The computational results have been obtained by generating random instances with the following characteristics:

- set of frequencies: $1,1 / 2,1 / 3,1 / 4,1 / 5,1 / 6,1 / 7,1 / 8,1 / 9,1 / 10$;

- number of products: $1000,5000,10000$;

- quantity per product per unit time $\left(q_{i}\right)$ : randomly generated between 0.1 and 5 , between 5 and 100 and between 0.1 and 100 ;

- unit volume per product $\left(v_{i}\right)$ : randomly generated between 0.001 and 0.01 ;

- unit inventory cost per product $\left(h_{i}\right)$ : randomly generated between 0.001 and 0.05 , between 0.05 and 1 and between 0.001 and 1 ;

- transportation cost per trip (c): 300 .

The results are shown in Table I. Each row corresponds to 5 problem instances with the characteristics described in columns 1-3. Column 1 gives the number of products; Column 2 gives the range of the quantity per product and Column 3 the range of the inventory $\operatorname{cost} h_{i}$.

Columns 4-7 show the computational results obtained by the branch-and-bound proposed in Speranza and Ukovich (1991), referred as Old branch-and-bound; Column 4 gives the average number of visited nodes (the maximum number of possible nodes has been fixed in 30000 ); Column 5 gives the number of times we have obtained the optimal solution on 5 instances; Column 6 the node in which the optimal solution has been found (average on the 5 instances); finally, Column 7 gives the average CPU time (minutes and seconds). In columns 8-11 the computational results obtained by the new branch-and-bound, referred as New branch-and-bound, are shown as in columns 4-7.

The computational results show that the new branch-and-bound is more efficient than the one proposed in Speranza and Ukovich (1991), both in terms of number of visited nodes and CPU time. 
Table I Comparison of the branch-and-bound algorithms

\begin{tabular}{|c|c|c|c|c|c|c|c|c|c|c|}
\hline \multicolumn{3}{|c|}{ Parameters } & \multicolumn{4}{|c|}{ Old branch-and-bound } & \multicolumn{4}{|c|}{ New branch-and-bound } \\
\hline Prod. & Quant. & Inventory & Nodes & $\mathrm{N}$ & Opt. & Time & Nodes & $\mathrm{N}$ & Opt. & Time \\
\hline 1000 & $0.1-5$ & $0.001-0.05$ & 13784 & 3 & 1064 & 05.09 & 3431 & 5 & 1031 & 00.53 \\
\hline 1000 & $0.1-5$ & $0.05-1$ & 148 & 5 & 125 & 00.02 & 51 & 5 & 37 & 00.00 \\
\hline 1000 & $0.1-5$ & $0.001-1$ & 192 & 5 & 160 & 00.03 & 136 & 5 & 114 & 00.02 \\
\hline 1000 & $5-100$ & $0.001-0.05$ & 22290 & 3 & 4676 & 06.44 & 8411 & 5 & 2021 & 01.41 \\
\hline 1000 & $5-100$ & $0.05-1$ & 1363 & 5 & 431 & 00.26 & 410 & 5 & 185 & 00.06 \\
\hline 1000 & $5-100$ & $0.001-1$ & 3996 & 5 & 1899 & 01.23 & 1100 & 5 & 728 & 00.18 \\
\hline 1000 & $0.1-100$ & $0.001-0.5$ & 9274 & 4 & 1387 & 02.47 & 1697 & 5 & 589 & 00.22 \\
\hline 1000 & $0.1-100$ & $0.05-1$ & 949 & 5 & 221 & 00.18 & 227 & 5 & 47 & 00.03 \\
\hline 1000 & $0.1-100$ & $0.001-1$ & 1970 & 5 & 1123 & 00.39 & 644 & 5 & 392 & 00.10 \\
\hline 5000 & $0.1-5$ & $0.001-0.05$ & 15255 & 3 & 1175 & 16.37 & 6251 & 5 & 1344 & 03.55 \\
\hline 5000 & $0.1-5$ & $0.05-1$ & 3341 & 5 & 944 & 03.36 & 681 & 5 & 300 & 00.31 \\
\hline 5000 & $0.1-5$ & $0.001-1$ & 1321 & 5 & 1090 & 01.26 & 610 & 5 & 507 & 00.30 \\
\hline 5000 & $5-100$ & $0.001-0.05$ & 30000 & 0 & 0 & 34.28 & 19070 & 5 & 690 & 12.15 \\
\hline 5000 & $5-100$ & $0.05-1$ & 2070 & 5 & 434 & 01.58 & 249 & 5 & 117 & 00.14 \\
\hline 5000 & $5-100$ & $0.001-1$ & 24608 & 1 & 1223 & 26.56 & 4776 & 5 & 666 & 03.06 \\
\hline 5000 & $0.1-100$ & $0.001-0.05$ & 30000 & 0 & 0 & 34.41 & 13097 & 5 & 645 & 08.33 \\
\hline 5000 & $0.1-100$ & $0.05-1$ & 2212 & 5 & 305 & 02.08 & 364 & 5 & 122 & 00.18 \\
\hline 5000 & $0.1-100$ & $0.001-1$ & 24798 & 1 & 92 & 27.08 & 6588 & 5 & 368 & 04.68 \\
\hline 10000 & $0.1-5$ & $0.001-0.05$ & 30000 & 0 & 0 & 59.02 & 10454 & 5 & 1730 & 12.01 \\
\hline 10000 & $0.1-5$ & $0.05-1$ & 3097 & 5 & 683 & 06.17 & 854 & 5 & 211 & 01.13 \\
\hline 10000 & $0.1-5$ & $0.001-1$ & 2562 & 5 & 689 & 05.13 & 850 & 5 & 345 & 01.14 \\
\hline 10000 & $5-100$ & $0.001-0.05$ & 4079 & 5 & 1306 & 07.18 & 584 & 5 & 515 & 00.52 \\
\hline 10000 & $5-100$ & $0.05-1$ & 3388 & 5 & 217 & 05.52 & 244 & 5 & 75 & 00.24 \\
\hline 10000 & $5-100$ & $0.001-1$ & 3615 & 5 & 794 & 06.21 & 418 & 5 & 348 & 00.39 \\
\hline 10000 & $0.1-100$ & $0.001-0.05$ & 4722 & 5 & 1954 & 08.42 & 803 & 5 & 577 & 01.10 \\
\hline 10000 & $0.1-100$ & $0.05-1$ & 3917 & 5 & 377 & 07.00 & 494 & 5 & 157 & 00.45 \\
\hline 10000 & $0.1-100$ & $0.001-1$ & 4725 & 5 & 1995 & 08.43 & 804 & 5 & 649 & 01.10 \\
\hline
\end{tabular}

\section{REFERENCES}

Anily, S. and Federgruen, A. (1990) One Warehouse Multiple Retailer Systems with Vehicle Routing Costs. Management Science, 36, 92-114.

Bertazzi, L., Speranza, M.G. and Ukovich, W. (1994) Minimization of logistic costs with given frequencies. Technical Report 79, Department of Quantitative Methods, University of Brescia (submitted).

Bertazzi, L., Speranza, M.G. and Ukovich, W. (1995) Dominance Rules and Approximate Solutions for the Transportation Problem with Discrete Available Frequencies, (in preparation).

Blumenfeld, D.E., Burns, L.D., Diltz, J.D. and Daganzo, C.F. (1985) Analyzing tradeoffs between transportation, inventory and production costs on freight networks. Transportation Research, 19B 361-80. 
Burns, L.D., Hall, R.W., Blumenfeld, D.E. and Daganzo, C.F. (1984) Distribution Strategies that Minimize Transportation and Inventory Cost. Operations Research, 33, $469-90$.

Keeney, R. L., and Raiffa, H. (1976) Decisions with multiple objectives. Wiley, New York. Speranza, M.G. and Ukovich, W. (1991) A capacitated transportation problem with factoring costs. Technical Report 26, Department of Quantitative Methods, University of Brescia (submitted).

Speranza, M.G. and Ukovich, W. (1994) Minimizing transportation and inventory costs for several products on a single link. Operations Research, 42, 879-94. 